\title{
Impact of the COVID-19 pandemic on blood pressure control: a nationwide home blood pressure monitoring study
}

\author{
Fabiana G. A. M. Feitosa ${ }^{1,2,3}$ - Audes D. M. Feitosa ${ }^{1,2,4}$ - Annelise M. G. Paiva ${ }^{5}$ Marco A. Mota-Gomes ${ }^{5}$. \\ Weimar S. Barroso ${ }^{6} \cdot$ Roberto D. Miranda $^{7}$ Eduardo C. D. Barbosa ${ }^{8}$. Andréa A. Brandão ${ }^{9}$ José L. Lima-Filho ${ }^{1}$. \\ Andrei C. Sposito ${ }^{10}$. Antonio Coca ${ }^{11} \cdot$ Wilson Nadruz Jr ${ }^{1,10}$
}

Received: 11 July 2021 / Revised: 15 August 2021 / Accepted: 30 September 2021 / Published online: 2 December 2021

(c) The Japanese Society of Hypertension 2021

\begin{abstract}
There are concerns that hypertension control may decrease during the COVID-19 pandemic. This study evaluated the impact of the COVID-19 pandemic on office blood pressure (OBP) and home blood pressure monitoring (HBPM) control in a large Brazilian nationwide sample. The results of an adjusted spline analysis evaluating the trajectory of OBP and HBPM control from 01/Jan/2019 to 31/Dec/2020 among independent participants who were untreated $(n=24,227)$ or treated $(n=27,699)$ with antihypertensive medications showed a modest and transient improvement in OBP control among treated individuals, which was restricted to the early months following the COVID-19 pandemic outbreak. Furthermore, slight reductions in OBP and HBPM values were detected in the early months following the COVID-19 pandemic outbreak among treated $(n=$ 987) participants for whom blood pressure measurements before and during the pandemic were available, but not among untreated $(n=495)$ participants. In conclusion, we found no major adverse influence of the COVID-19 pandemic on OBP and HBPM control in a large nationwide sample.
\end{abstract}

Keywords Home blood pressure $\cdot$ Office blood pressure $\cdot$ COVID-19 $\cdot$ Blood pressure control $\cdot$ Hypertension

\section{Introduction}

The coronavirus disease 2019 (COVID-19) pandemic is expected to have adverse effects on the long-term incidence

Supplementary information The online version contains supplementary material available at https://doi.org/10.1038/s41440021-00784-1.

Wilson Nadruz

wilnj@fcm.unicamp.br

1 Laboratory of Immunopathology Keizo Asami, Federal University of Pernambuco, Recife, PE, Brazil

2 Pronto Socorro Cardiológico de Pernambuco (PROCAPE), University of Pernambuco, Recife, PE, Brazil

3 University Hospital Oswaldo Cruz, University of Pernambuco, Recife, PE, Brazil

4 UNICAP Clinical Research Institute, Recife, PE, Brazil

5 CESMAC University Center/Heart Hospital of Alagoas, Maceió, AL, Brazil

6 Hypertension League, Federal University of Goiás, Goiânia, GO, Brazil of cardiovascular diseases due to inadequate control of cardiovascular risk factors [1]. In particular, there are concerns that hypertension control may decrease during the pandemic due to prolonged stress and unfavorable lifestyle habits, including increased alcohol and salt intake, weight gain and sedentarism [1]. The lack of hypertension control might also be relevant in the context of SARS-CoV-2 infection, since worse blood pressure (BP) control has been

7 Cardiovascular Section, Geriatrics Division, Federal University of São Paulo, São Paulo, SP, Brazil

8 Department of Hypertension and Cardiometabolism, São Francisco Hospital - Santa Casa de Porto Alegre, Porto Alegre, Brazil

9 School of Medical Sciences, State University of Rio de Janeiro, Rio de Janeiro, RJ, Brazil

10 Department of Internal Medicine, School of Medical Sciences, State University of Campinas, SP Paulo, Brazil

11 Hypertension and Vascular Risk Unit, Department of Internal Medicine, Hospital Clínic, University of Barcelona, Barcelona, Spain 


\section{Graphical Abstract}

*Adjusted prevalence of high office blood pressure (OBP) and home blood pressure monitoring (HBPM) before and during the Covid-19 pandemic among independent treated participants. ${ }^{* *} p<0.05$. Whisker-plot: $95 \%$ confidence interval.

Treated participants with single HBPM exams $(n=27,699)$
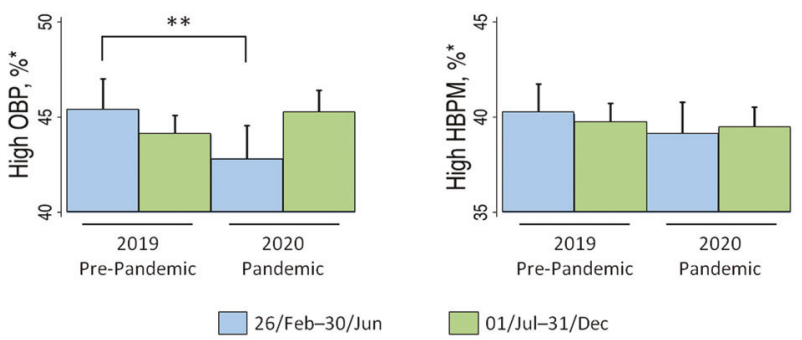

associated with adverse outcomes among patients with COVID-19 [2, 3].

Conflicting data have been reported regarding the impact of the pandemic on hypertension control, since both shortterm increases $[4,5]$ and decreases $[6,7]$ in BP levels have been reported following the pandemic outbreak. Furthermore, little is known regarding the medium/long-term impact of the pandemic on BP levels and BP control for the population. This study evaluated the impact of the COVID-19 pandemic on office BP (OBP) and home BP monitoring (HBPM) control in a large Brazilian nationwide sample that was followed until December 2020.

\section{Methods}

The first COVID-19 case was diagnosed in Brazil on 26/Feb/2020, and within one month, all Brazilian states had been severely affected by the pandemic [8]. We performed two independent analyses using data from 57,768 individuals aged $>18$ years who underwent OBP and HBPM measurement between May 2017 and December 2020 at 719 centers located in 26 of the 27 Brazilian states using an online platform (telemrpa.com.br) [9-12]. The first set of analyses evaluated independent participants who were untreated $(n=24,227)$ or treated $(n=27,699)$ with antihypertensive medications and had one HBPM value available from 01/Jan/2019 to 31/Dec/2020 (Cohort 1). The second set of analyses evaluated 495 untreated and 987 treated participants who had BP measurements available from before and during the pandemic (Cohort-2). OBP was calculated as the average of two office readings, and HBPM was calculated as the average of three home BP measurements taken in the morning and in the evening for 4 consecutive days using validated devices (HEM-705CP, HEM-7113, HEM-7320 or HEM-9200T; Omron Health Care, Japan), as previously described [9-12]. High OBP

was defined as an office systolic $\mathrm{BP} \geq 140 \mathrm{mmHg}$ or diastolic $\mathrm{BP} \geq 90 \mathrm{mmHg}$, while high HBPM was defined as a home systolic $\mathrm{BP} \geq 135 \mathrm{mmHg}$ or diastolic $\mathrm{BP} \geq 85 \mathrm{mmHg}$ [13]. The protocol was approved by the Oswaldo Cruz University Hospital/PROCAPE Ethics Committee.

Continuous and categorical variables are presented as the mean \pm standard deviation and proportion. The trajectory of OBP and HBPM control among Cohort-1 participants was assessed by restricted cubic splines with seven knots (unadjusted and adjusted for age, sex, body mass index, center and the average monthly temperature of the state where the center was located). Logistic regression analysis adjusted for the same variables compared the prevalence of high OBP and HBPM before and after the pandemic among Cohort-1 participants. Differences in continuous and categorical variables before and after the pandemic among Cohort-2 participants were evaluated by using the paired $t$-test and McNemar's test, respectively. $P$ values $<0.05$ were considered significant. Statistical analysis was performed using Stata 14.1 (Stata Corp LP, College Station, TX, USA).

\section{Results}

Among untreated and treated individuals in Cohort-1, the number of HBPM exams decreased markedly after the diagnosis of the first COVID-19 case, reached a nadir in April 2020 and tended to return to pre-pandemic levels after June 2020 (Fig. 1). For this reason, we divided the pandemic period into early (from $26 / \mathrm{Feb} / 2020$ to $30 / \mathrm{Jun} / 2020$ ) and late (from 01/Jul/2020 to 31/Dec/2020) periods. The results of unadjusted (Supplemental Fig. 1) and adjusted (Fig. 1) spline analysis showed a seasonal inverted $\mathrm{U}$-shaped trajectory for the prevalence of high OBP and HBPM across the years 2019 and 2020 among untreated and treated participants. However, the trajectories suggested 


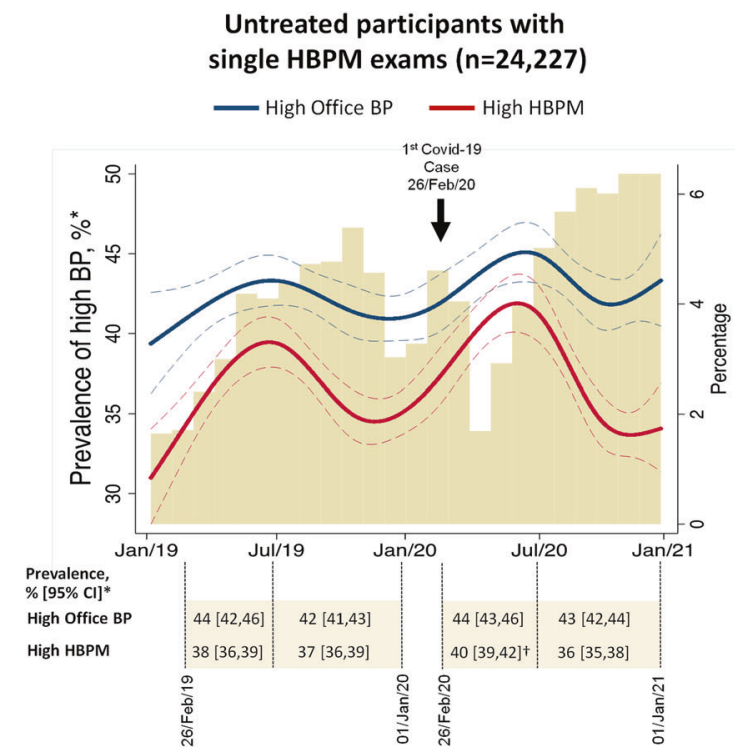

Fig. 1 Impact of the COVID-19 pandemic on the trajectories of high office BP and HBPM. Adjusted restricted cubic splines for the relationship between the prevalence of high office BP and high HBPM and calendar time among independent participants for whom a single HBPM exam was reported from 01/Jan/202019 to 31/Dec/2020. High office BP (blue lines) was defined as an office systolic $\mathrm{BP} \geq 140 \mathrm{mmHg}$ or diastolic $\mathrm{BP} \geq 90 \mathrm{mmHg}$, while high home BP (red lines) was defined as a home systolic $\mathrm{BP} \geq 135 \mathrm{mmHg}$ or diastolic $\mathrm{BP} \geq 85 \mathrm{mmHg}$. The prevalence of high office and home BP was calculated on a daily

a trend toward a higher prevalence of high OBP and HBPM among untreated participants and a lower prevalence of high $\mathrm{OBP}$ and HBPM among treated participants during the early pandemic period compared with the corresponding period in 2019. These trends resulted in a modestly greater adjusted prevalence of high HBPM among untreated participants (adjusted prevalence [95\% confidence interval] $=40$ $[39,42] \%$ vs. $38[36,39] \% ; p=0.025)$ and a lower prevalence of high OBP among treated participants (adjusted prevalence $[95 \%$ confidence interval $]=43[41,44] \%$ vs. 45 $[44,47] \% ; p=0.032$ ) when comparing the $26 / \mathrm{Feb} / 2020-30 /$ Jun/2020 and 26/Feb/2019-30/Jun/2019 periods (Fig. 1).

To evaluate the Cohort-2 participants, we initially divided the sample into those with repeated BP measurements during the early (from $26 / \mathrm{Feb} / 2020$ to $30 / \mathrm{Jun} / 2020$ ) or late (from $01 / \mathrm{Jul} / 2020$ to $31 / \mathrm{Dec} / 2020$ ) pandemic periods and then compared the BP characteristics before and during the pandemic (Table 1). In general, there were no significant differences in BP values or rates of high BP before and during the pandemic, except for the presence of lower OBP and HBPM levels during the early pandemic period among treated participants (Table 1). Furthermore, in a subsample of treated Cohort-2 participants for whom data on antihypertensive medication use were available, there was no difference in the use of antihypertensive medications before and during the pandemic, although lower OBP and HBPM

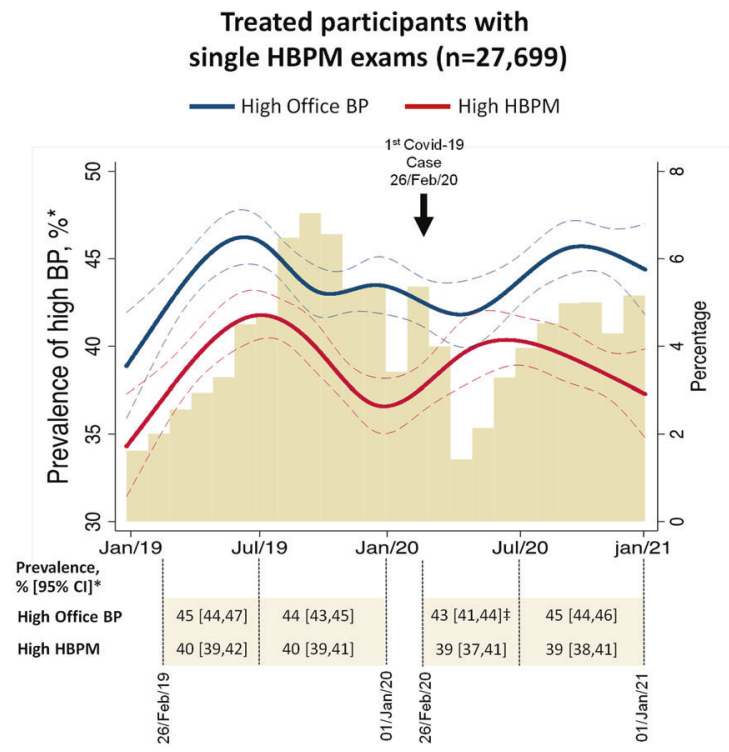

basis. The dashed lines indicate the $95 \%$ confidence intervals. The brown bars are histograms of the distribution (in percentage) of HBPM exams per month. ${ }^{*}$ adjusted for age, sex, body mass index, center and the average monthly temperature of the state where each center was located. ${ }^{\dagger} p=0.025$ compared with high HBPM prevalence during the period from 26/Feb/2019 to 30/Jun/2019 among untreated participants. ${ }^{+} p=0.032$ compared with high OBP prevalence during the period from 26/Feb/2019 to 30/Jun/2019 among treated participants. BP-blood pressure; HBPM-home BP monitoring; $\mathrm{CI}$-confidence interval

levels were observed during the early pandemic period (Supplemental Table 1).

\section{Discussion}

This report showed a modest and transient improvement in BP levels among treated hypertensive individuals in both Cohorts 1 and 2 that was restricted to the early months after the COVID-19 pandemic outbreak. These findings agree with European and Chinese data suggesting that HBPM values were lower among hypertensive individuals during the early weeks of the COVID-19 outbreak than during the pre-pandemic period $[6,7]$. The mechanisms underlying these findings are not clear, but it is possible that psychological and physical relaxation related to mobility restrictions and lockdowns imposed during the pandemic outbreak might have overcome COVID-19-related stressors, at least among treated hypertensive individuals [6, 7]. Conversely, we found a slight and transient worsening of HBPM control during the early phases of the pandemic among untreated individuals in Cohort-1, but these results were not reproduced in untreated individuals in Cohort-2, which might limit the consistency of these findings.

A major novel finding of this report was that there were no significant differences in OBP and HBPM during the 


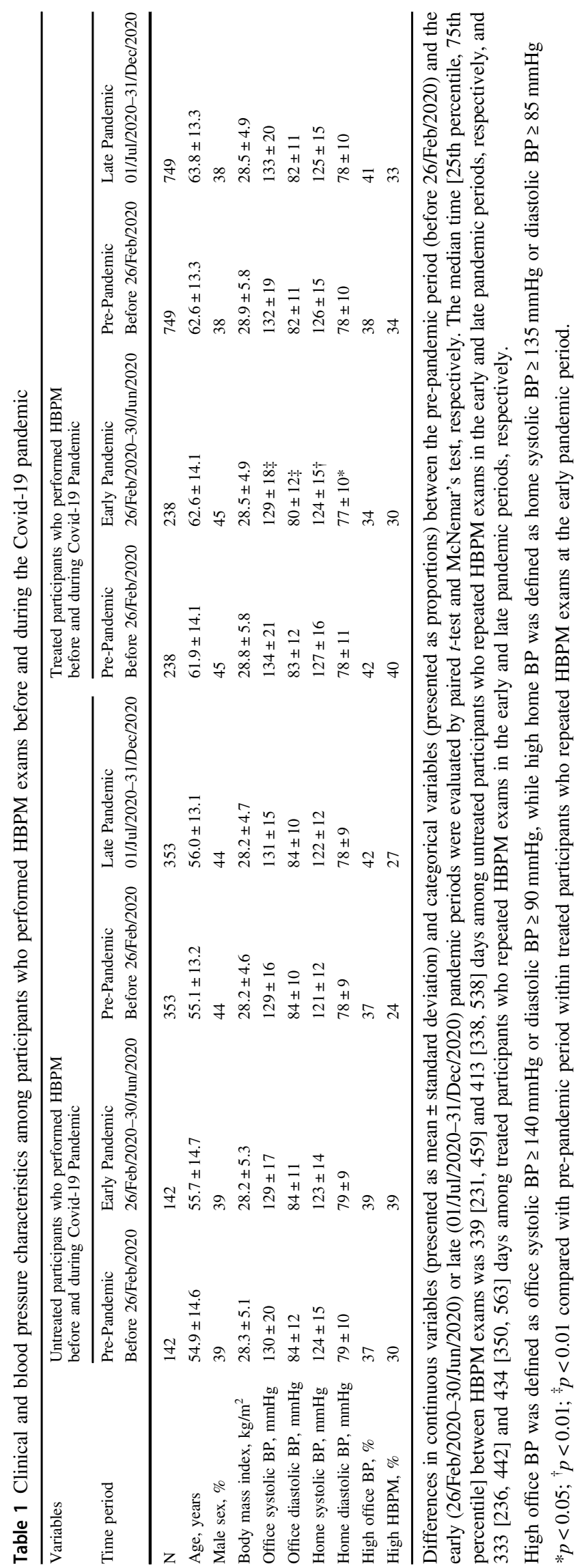

second semester of 2020 compared with the second semester of 2019 among either treated or untreated individuals in Cohorts 1 and 2. These data suggest that the pandemic did not have a major medium-term impact on BP control, although the patients may have experienced prolonged stress and adopted unfavorable lifestyle habits [1]. Because hypertension and worse $\mathrm{BP}$ control are reported to be risk factors for a worse prognosis following SARS-CoV-2 infection $[2,3,14]$, it is also possible that the patients may have been more worried about controlling their BP, which could explain the lack of an adverse impact of the pandemic on BP control.

This study has some limitations. First, the possibility that selection bias influenced our results cannot be discarded, since patients who sought $\mathrm{BP}$ evaluation during the pandemic may have been cautious about their health than those who did not and thus may have had better BP control. Second, information on additional risk factors for hypertension and cardiovascular diseases was not available. Conversely, the nationwide multicenter design, large sample size and extended period of observation were strengths of our report.

In conclusion, we found no major adverse influence of the COVID-19 pandemic on OBP and HBPM control in a large nationwide sample. Ongoing evaluation and surveillance are necessary to assess whether the COVID-19 pandemic may lead to adverse hypertension control and an increased risk of future cardiovascular events.

Funding The study was supported by a grant from the Brazilian National Council for Scientific and Technological Development (CNPq; grant 306154/2017-0) to Dr WN-Jr.

\section{Compliance with ethical standards}

Conflict of interest ADMF, MAM-G, WSB, AAB, RDM, and ECDB are owners of the online TELEMRPA platform (Beliva, Brazil). ADMF, MAM-G, and WSB are consultants for Omron.

Publisher's note Springer Nature remains neutral with regard to jurisdictional claims in published maps and institutional affiliations.

\section{References}

1. European Society of Hypertension Corona-virus Disease 19 Task Force. The corona-virus disease 2019 pandemic compromised routine care for hypertension: a survey conducted among excellence centers of the European Society of Hypertension. J Hypertens. 2021;39:190-5.

2. Ran J, Song Y, Zhuang Z, Han L, Zhao S, Cao P, et al. Blood pressure control and adverse outcomes of COVID-19 infection in patients with concomitant hypertension in Wuhan, China. Hypertens Res. 2020;43:1267-76.

3. Geng L, He C, Kan H, Zhang K, Mao A, Zhang C, et al. The association between blood pressure levels and mortality in critically ill patients with COVID-19 in Wuhan, China: a case-series report. Hypertens Res. 2021;44:368-70. 
4. Zhang S, Zhou X, Chen Y, Wang L, Zhu B, Jiang Y, et al. Changes in Home Blood Pressure Monitored Among Elderly Patients With Hypertension During the COVID-19 Outbreak: A Longitudinal Study in China Leveraging a SmartphoneBased Application. Circ Cardiovasc Qual Outcomes. 2021;14: e007098.

5. Fosco MJ, Silva P, Taborda GA, Ahumada L. Association between mandatory lockdown due to COVID-19 and severe arterial hypertension. Medicina 2020;80:25-9.

6. Pengo MF, Albini F, Guglielmi G, Mollica C, Soranna D, Zambra $\mathrm{G}$, et al. Home blood pressure during COVID-19-related lockdown in patients with hypertension. Eur J Prev Cardiol. 2021. https://doi.org/10.1093/eurjpc/zwab010.

7. Girerd N, Meune C, Duarte K, Vercamer V, Lopez-Sublet M, Mourad JJ. Evidence of a Blood Pressure Reduction During the COVID-19 Pandemic and Associated Lockdown Period: Insights from e-Health Data. Telemed J E Health. 2021. https://doi.org/10. 1089/tmj.2021.0006.

8. Coronavirus Brazil. https://www.worldometers.info/coronavirus/ country/brazil/.

9. Feitosa ADM, Mota-Gomes MA, Barroso WS, Miranda RD, Barbosa ECD, Pedrosa RP, et al. Blood pressure cutoffs for white-coat and masked effects in a large population undergoing home blood pressure monitoring. Hypertens Res. 2019;42: 1816-23.

10. Feitosa ADM, Mota-Gomes MA, Barroso WS, Miranda RD, Barbosa ECD, Pedrosa RP, et al. Relationship between office isolated systolic or diastolic hypertension and white-coat hypertension across the age spectrum: a home blood pressure study. J Hypertens. 2020;38:663-70.

11. Feitosa ADM, Mota-Gomes MA, Barroso WS, Miranda RD, Barbosa ECD, Pedrosa RP, et al. Correlation between office and home blood pressure in clinical practice: a comparison with 2017 American College of Cardiology/American Heart Association Hypertension Guidelines recommendations. J Hypertens. 2020;38:179-81.

12. Feitosa ADM, Mota-Gomes MA, Barroso WS, Miranda RD, Barbosa ECD, Brandão AA, et al. The impact of changing home blood pressure monitoring cutoff from $135 / 85$ to $130 / 80 \mathrm{mmHg}$ on hypertension phenotypes. J Clin Hypertens. 2021;23:1447-51.

13. Williams B, Mancia G, Spiering W, Agabiti Rosei E, Azizi M, Burnier M, et al. ESC/ESH Guidelines for the management of arterial hypertension. J Hypertens. 2018;2018:1953-2041.

14. Guan WJ, Ni ZY, Hu Y, Liang WH, Ou CQ, He JX, et al. Clinical Characteristics of Coronavirus Disease 2019 in China. N Engl J Med. 2020;382:1708-20. 